\title{
RÉVOLUTION ET UTOPIE DANS LE ROMAN LE SIÈCLE DES LUMIĖRES D'ALEJO CARPENTIER (1958) : LES ITINÉRAIRES D'UN DÉSENCHANTEMENT
}

UDC 821.134.2(729.1).09-31 Carpentier A.

\author{
Jean-Jacques Tatin-Gourier ${ }^{1}$, Dragan Bogojević ${ }^{2}$ \\ ${ }^{1}$ Université de Tours, Interactions culturelles et discursives, Tours, France, \\ ${ }^{2}$ Université du Monténégro, Faculté de philologie, Nikšić, Monténégro
}

\begin{abstract}
Résumé. Des répercussions de la Révolution française aux Caraïbes - abolition et restauration de l'esclavage - à la terreur de la France en révolution et à l'Espagne occupée par les troupes de Napoléon le couple de jumeaux que constituent Sofia et Esteban parcourt une à une les étapes d'une désillusion et d'un dépouillement absolus avant de disparaître à Madrid dans les hécatombes du Tres de Mayo dont Goya - constamment cité dans le roman - a été le grand témoin. Les chemins de l'utopie de la jeunesse et de l'espoir débouchent ainsi sur une apocalypse. En nous appuyant notamment sur les travaux de l'historien des imaginaires sociaux Bronislaw Baczko et en analysant plus précisément les mutations des personnages dans leur confrontation aux événements successifs, nous nous proposons de mettre en lumière cette dynamique de la désillusion.
\end{abstract}

Mots-clés : révolution française, esclavage, utopie, désillusion, France, île.

\section{INTRODUCTION}

Dans son roman Le Siècle des Lumières publié en 1958 et ancré dans les colonies espagnoles et françaises des Caraïbes à la fin du XVIII ${ }^{\mathrm{e}}$ siècle (de Cuba à Saint-Domingue, de la Guadeloupe à la Guyane) mais aussi dans une Europe bouleversée par la Révolution (du Paris de la Terreur au Madrid de l'insurrection contre l'occupation française en 1808), Alejo Carpentier a en fait avant tout envisagé les inflexions des imaginaires utopiques au cœur d'une révolution qui se heurte à l'ordre esclavagiste des «îles», qui prétend conjuguer libération et combat sans concession les «contres révolutionnaires » pour finalement refluer vers une restauration sociale et religieuse que rien ne laissait prévoir. Focalisé sur le devenir du couple central du roman (Sofia et son cousin Esteban) guidé par le révolutionnaire français Victor Hugues (figure historique attestée), le roman met en scène

Submitted July 3, 2019; Accepted September 14, 2019

Corresponding author: Jean-Jacques Tatin-Gourier

Université de Tours, Interactions culturelles et discursives, Tours, France

E-mail: tatin-gourier@univ-tours.fr 
et individualise ces inflexions qui conduisent les deux jeunes gens, à des rythmes différents et nettement décalés, du rêve utopique à l'action révolutionnaire, de cet engagement à une désillusion qui, en tout cas pour Sofia, coïncide clairement avec le constat désolé du rétablissement de l'esclavage ordonné par Bonaparte premier consul.

Une désillusion dont l'ultime et mortel horizon est, pour Sofia et Esteban, le massacre du Tres de Mayo 1808 à Madrid, massacre illustré par le grand tableau de Goya et dans lequel disparaît à jamais le couple qui a parcouru le grand arc des révolutions de la fin du XVIII ${ }^{\mathrm{e}}$ siècle.

Nous nous proposons ici, dans le cadre d'une étude des deux protagonistes (tout en tenant compte du système global des personnages du roman tel que l'a notamment entendu Philippe Hamon (Hamon 1972, 86-110) d'analyser les mises en scène romanesques de la désillusion de l'utopie révolutionnaire.

Nous nous interrogerons enfin sur le caractère pionnier de la réflexion sur l'utopie qui sous-tend l'écriture du roman Le Siècle des Lumières. Caractère pionnier : ce n'est que dans les années 1980 que des historiens des idées et de la culture (c'est notamment le cas de Bronislaw Baczko avec son ouvrage Lumières de l'utopie) ont analysé les imaginaires utopiques et leurs dynamiques, en privilégiant eux aussi le second versant du XVIII siècle et la Révolution française.

\section{REVOLUTION, AUBE D’UN MONDE NOUVEAU}

Les premières séquences du roman mettent en scène une fratrie (Carlos, l'aîné et qui sera le seul survivant, Sofia et leur cousin orphelin Esteban) que la mort d'un père grand commerçant de La Havane a libéré de toute entrave ${ }^{1}$.

Dans la grande maison coloniale, pourvue d'entrepôts attenants, de multiples rêves se déploient. Dans ces rêves dont le narrateur souligne le plus souvent le caractère naïf, le Paris des Lumières, des lettres et des arts occupe une place centrale : «Esteban rêvait de Paris et de ses expositions de peinture, de ses cafés intellectuels, de sa vie littéraire ; il voulait suivre un cours de ce fameux Collège de France où l'on enseignait des langues orientales dont l'étude, sinon très utile pour gagner de l'argent, devait être passionnante pour celui qui aspirait comme lui à lire directement sur les manuscrits des textes asiatiques récemment découverts. Pour Sofia il y avait les représentations de l'Opéra et du Théâtre Français, dans le vestibule duquel on pouvait admirer quelque chose d'aussi beau et d'aussi réputé que le Voltaire de Houdon. »(Carpentier 1962, 36-37). Dans la maison-labyrinthe interviennent aussi divers appareils de physique - qui semblent autant d'échos des planches de l'Encyclopédie - et une multiplicité de livres, une véritable « bibliothèque d'idées et de poésies nouvelles ». (Carpentier 1962, 39)

C'est dans cet étrange espace que fait mystérieusement irruption, Victor Hugues, personnage dont la dimension historique est attestée, négociant venu de Port-au-Prince, capitale de la colonie française de Saint-Domingue ${ }^{2}$. Ce Français, dynamique, sociable et

\footnotetext{
${ }^{1}$ Un bref prologue rédigé à la première personne, relate toutefois l'approche du navire qui conduit à Pointe à Pitre, sous la houlette du révolutionnaire français Victor Hugues, la guillotine. Aucun personnage n'est nommé dans ce prologue que la lecture ultérieure du roman permet d'attribuer à Esteban de retour dans les Caraïbes. Dans ce prologue, la focalisation sur la guillotine est en fait exclusive. Un bref prologue rédigé à la première personne, relate toutefois l'approche du navire qui conduit à Pointe à Pitre, sous la houlette du révolutionnaire français Victor Hugues, la guillotine.

${ }^{2}$ Victor Hugues (1762-1826) est né dans une famille de colons de Saint-Domingue. Il quitta l'île pour la France lors de la révolution haïtienne. Il devint, avec le soutien des jacobins, Procureur du Comité de salut public de La
} 
entreprenant éblouit la fratrie par son aisance, sa maîtrise des êtres et des choses, son sens $\mathrm{du}$ jeu, sa connaissance du monde et des livres. Il est aussi celui qui conduit auprès d'Esteban, atteint d'un terrible asthme chronique, le médecin Ogé ${ }^{3}-$ autre personnage dont le nom renvoie à l'histoire de la Révolution dans les îles - homme de couleur de Saint-Domingue, «philanthrope », franc-maçon tout comme son ami Victor Hugues qui se révèle en mesure de guérir Esteban. Victor Hugues, et à moindres égards Ogé, seront les vecteurs des idées nouvelles introductrices des perspectives révolutionnaires. Victor Hugues, de par l'influence qu'il acquiert très vite, notamment sur Esteban, sera le grand inspirateur, le maître à penser et à agir, voire, dans une vision plus pessimiste qui ne s'imposera qu'à la fin de l'aventure révolutionnaire, le grand manipulateur fauteur d'aliénation, de désenchantement et de malheur.

À La Havane, la rumeur, vite reprise par la police du roi d'Espagne, d'appartenance à la franc-maçonnerie, contraint Victor Hugues et Ogé à un départ précipité de Cuba. Alors que Carlos, moins menacé, demeure à Cuba pour gérer les entrepôts de l'entreprise familiale, Esteban et Sofia embarquent aux côtés de Victor Hugues et d'Ogé. Pour Esteban - Sofia demeure dans les Caraïbes - le grand voyage qui commence vers la France aux côtés de Victor Hugues sera dans un premier temps une découverte progressive et enthousiaste d'une révolution perçue comme aube d'un monde nouveau. Avant même qu'elle ne décide de demeurer dans les «îles», Sofia est elle-même convaincue et enthousiasmée : «Deux jours se passèrent à parler de révolutions, Sofia était étonnée de voir à quel point le nouveau sujet de conversation était passionnant pour elle. Parler de révolutions, imaginer des révolutions, se situer mentalement au sein d'une révolution, c'est se rendre un peu mentalement au sein d'une révolution, c'est se rendre un peu maître du monde. Ceux qui parlent d'une révolution se voient poussés à la faire. Il est si évident que tel ou tel privilège doit être aboli, que l'on procède à son abolition. Il est si certain que telle oppression est odieuse que l'on édicte des mesures contre elle ; il est si clair que tel personnage est un misérable, qu'on le condamne à mort à l'unanimité. Et une fois le terrain assaini, on entreprend d'édifier la cité de l'avenir. » (Carpentier 1962, 102-103)

Un bref aperçu de Saint-Domingue dévastée par le soulèvement des esclaves (Victor Hugues et Esteban réembarquent immédiatement pour la France) n'hypothèque nullement l'enthousiasme pour la révolution en cours. La traversée de l'Atlantique est présentée en termes révélateurs : elle rappelle en effet à Esteban la marche de Moïse guidant son peuple vers Canaan : «Vers l'orient se dressait toute droite, magnifique, aperçue par les yeux de l'intelligence, la colonne de feu qui guide les marches vers toute terre promise.» (Carpentier 1962, 128)

La France - et d'abord Paris, cœur de la Révolution - sera donc pour Esteban l'utopie en cours de construction, terre de liberté, de justice et préfiguration du bonheur humain à venir. Du même coup Victor Hugues, son tuteur et mentor, est en quelque sorte le prophète et le législateur en puissance de cette utopie : le Moïse des temps nouveaux. Esteban inscrit de plus son voyage et son aventure dans un cadre interprétatif dominé par deux modèles narratifs qui tendent à se superposer et à se confondre : celui de l'utopie et celui de l'épopée : «Il comprenait désormais le sens exact de son hallucinante traversée, semblable à celle de Perceval à la recherche de lui-même, vers la cité de l'avenir qui pour

Rochelle. Gouverneur de la Guadeloupe, il supervisa l'application du décret d'abolition de l'esclavage. Gouverneur de la Guyane en 1799, il remit en place l'esclavage sous le consulat. Il redevint gouverneur de la Guyane sous la Restauration (1817).

${ }^{3}$ Le Frère d'Ogé - dirigeant des hommes de couleur libres de Saint-Domingue, condamné au supplice de la roue et exécuté à son retour de France. 
une fois n'était pas située en Amérique comme celle de Thomas More ou de Campanella, mais au berceau même de la philosophie. » (Carpentier 1962, 138)

Le narrateur insiste sur la métamorphose du comportement d'Esteban devenu parisien. Surnommé le Huron en souvenir de l'Ingénu de Voltaire, le jeune homme déambule avec ferveur dans le Paris bouleversé de la Révolution, fréquente les étrangers - et notamment les Espagnols - venus à Paris pour partager l'enthousiasme révolutionnaire, est initié à la franc-maçonnerie dans la Loge des Etrangers Réunis. Esteban est littéralement métamorphosé : il adopte en effet un comportement marqué par la violence et la brutalité qui lui semblent adaptées à son engagement au service d'une Révolution qui se doit elle-même d'être violente et brutale : «(Il) faisait tout son possible pour heurter les habitudes de courtoisie de l'ancien régime, affichant une franchise, une brutalité verbale, une dureté dans ses jugements, qui blessaient parfois les révolutionnaires eux-mêmes. » (Carpentier 1962, 134)

Une première relégation dysphorique va toutefois intervenir pour Esteban. Sur la consigne de Victor Hugues, il est en effet envoyé à la frontière espagnole du Pays Basque pour écrire et traduire les textes visant à étendre la révolution à l'Espagne. Alors que Victor Hugues se rapproche des jacobins les plus radicaux, «fraye » avec Billaud-Varenne et Collot d'Herbois, professe la plus grande admiration pour Robespierre et entame par là-même une marche vers le pouvoir qui le conduira à l'exercice d'une terreur particulièrement cruelle ${ }^{4}$.

Esteban, éloigné, se découvre relégué, réduit à des tâches bureaucratiques et incapable de comprendre, tout comme l'est la population basque, les retournements de la situation politique parisienne. Témoin d'un exercice provincial, dérisoire et sordide de la Terreur avant même qu'il n'encoure lui-même de véritables dangers en tant qu'étranger, Esteban est en proie à une irrépressible tristesse même s'il pense encore, à l'inverse de ses compagnons espagnols, que « l'humanité sortira régénérée de ce bain de sang ». (Carpentier 1962, 155). Très vite, cependant, s'ajoute à cela le sentiment croissant de menaces liées à son statut d'étranger. Esteban trouve l'occasion propice pour quitter un pays dominé par une peur irrépressible pour tous ${ }^{5}$. C'est à nouveau sous la houlette de Victor Hugues, chargé par le Comité de salut public de s'assurer du contrôle de la Guadeloupe, qu'Esteban va à nouveau, mais en sens inverse, traverser l'Atlantique.

Désormais et pour l'ample séquence qui suivra, de l'embarquement sur le navire La Borée à la Guadeloupe et à la Guyane, Esteban est à nouveau en contact avec Victor Hugues. Il est désormais en mesure de mieux comprendre la grande ambiguïté qui caractérise un personnage qui, dans cette nouvelle aventure, est en effet chargé d'apporter en Guadeloupe le décret d'abolition de l'esclavage et transporte avec lui la guillotine dont il ne tardera pas à assurer un exercice massif. Et c'est précisément l'expérience de cette ambiguïté qui conduira Esteban à perdre toute illusion à la fois sur Victor Hugues, qui fut son «éclaireur» et son «guide» et plus globalement sur l'horizon prétendu de la Révolution, la cité juste, libre et heureuse appelée à devenir un modèle pour l'humanité tout entière. Cette désillusion, déjà amorcée lors du séjour d'Esteban aux marches d'Espagne, va se renforcer avec, en Guadeloupe, la banalisation du grand spectacle de mort de la guillotine : «Ce jour-là vit le commencement de la grande terreur dans l'île. La machine ne s'arrêtait plus de fonctionner sur la place de la Victoire, accélérant le rythme de ses coups.

\footnotetext{
${ }^{4}$ «(Esteban) savait qu'il remplissait de terrible façon la fonction d'accusateur public devant le tribunal révolutionnaire de Rochefort. Il était allé jusqu'à demander, chose qu'approuvait le jeune homme, que la guillotine fût installée dans la salle même des tribunaux, pour qu'on ne perdît pas de temps entre l'arrêt et l'exécution. » (Carpentier 1962, 151-152)

5 «Une grande peur commençait à inquiéter les nuits des habitants de cette côte. De nombreux regards surveillaient des rues derrière les volets entrebâillés des maisons plongées dans les ténèbres. » (Carpentier 1962, 158-159)
} 
Et comme on était fort curieux d'assister aux exécutions, en une ville où tout le monde se connaissait de vue ou se fréquentait (un tel n'oubliait pas la rancune qu'il portait à tel autre, ou une humiliation infligée), la guillotine centralisa désormais la vie de la cité.» (Carpentier 1962, 208)

Avec la nouvelle - nécessairement différée dans les Caraïbes - de la journée du 9 thermidor et de la chute de Robespierre, Victor Hugues maintient dans un premier temps et apparemment son adhésion aux valeurs jacobines (il se lancera en fait très vite dans la guerre de course et il saura ultérieurement, en Guyane, composer avec les politiques successives du Directoire et du Consulat). Esteban pour sa part est désormais animé par l'espoir d'un accès à une sorte de retour du droit, tout en refusant résolument une restauration monarchique : «Quelque chose de juste pouvait encore surgir ; de plus juste peut-être que ce qui tant de fois avait cessé de l'être parce que « on avait trop parlé c'avait été l'un des maux de l'époque - en termes abstraits. » (Carpentier 1962, 214-215)

\section{DESILLUSIONS À L'EGARD DE L'UTOPIE REVOLUTIONNAIRE}

Esteban est extrêmement sensible au fait de plus en plus fréquemment attesté que les esclaves que les esclaves prétendument libres depuis l'abolition officielle de l'esclavage sont en fait soumis désormais à un impitoyable travail forcé qui n'a rien à envier au Code noir de l'ancien régime : "Les nègres avaient été déclarés libres citoyens, mais ceux qui n'étaient pas soldats ou marins par la force courbaient le dos de l'aube au crépuscule comme autrefois, sous le fouet de leurs surveillants, derrière lesquels se dessinait par surcroît l'implacable azimut de la guillotine. » (Carpentier 1962, 224)

Horrifié, Esteban découvrira de plus très vite que Victor Hugues, dans la guerre de course qu'il organise en une sorte de fuite en avant, organise la vente des esclaves de la traite transportés dans les vaisseaux étrangers capturés : " Le commandant prit dans son bureau un pli d'instructions écrites de la propre main de Victor Hugues : «La France, en vertu de ses principes démocratiques, ne peut pas exercer la traite. Mais les commandants de navires corsaires sont autorisés, s'ils l'estiment convenable ou nécessaire, à vendre dans les ports hollandais les esclaves pris aux Anglais, aux Espagnols, et autres ennemis de la République. - Mais c'est infâme ! s'écria Esteban. Et nous avons aboli la traite pour servir de négriers parmi d'autres nations ?» (Carpentier 1962, 256-257)

De retour à La Havane auprès de Sofia et de Carlos, dans la grande maison familiale emplie de souvenirs - après son passage par la Guyane où il a notamment côtoyé BillaudVarenne, le dirigeant jacobin organisateur de la Terreur, déporté après le 9 thermidor, Esteban est un homme épuisé, absolument désenchanté, ayant perdu toute foi dans la dynamique révolutionnaire, tout espoir dans la cité utopique libre et juste de l'avenir : «(...) c'est vers un monde meilleur qu'était parti Esteban, il n'y avait pas si longtemps, ébloui par la grande colonne de feu qui semblait s'élever à l'Orient. Il revenait à présent de son voyage, frustré, ployant sous une énorme fatigue qui cherchait en vain un soulagement dans le souvenir de quelque aimable péripétie. A mesure que s'écoulait le temps de la traversée, il évoquait les aventures qu'il avait vécues tel un long cauchemar cauchemar traversé d'incendies, de persécutions, de châtiments - annoncé par le Cazotte dont les chameaux vomissaient des lévriers, par les nombreux augures de la fin des temps, qui avaient tant proliféré en ce siècle si long qu'il était aussi chargé d'actions que plusieurs siècles réunis. Les couleurs, les sons, les mots, qui le poursuivaient encore, provoquaient en lui un malaise profond, semblable à celui qu'engendrent en quelque 
point de la poitrine, là où les angoisses se font palpables sous forme de battements et d'asymétries, de rythmes viscéraux, les derniers soubresauts d'une maladie qui aurait pu être mortelle. Ce qui restait en arrière, évoqué dans des abîmes de ténèbres et de tumulte, au milieu des roulements de tambours et des râles d'agonie, des cris et des supplices, s'associait dans son esprit à l'idée de tremblement de terre, de convulsion collective, de fureur rituelle ... « Je viens de vivre parmi les barbares », dit Esteban à Sofia, lorsque pour lui s'ouvrit dans un solennel grincement de gonds la porte épaisse de la maison familiale toujours debout au même coin de rue, typiquement ornée de hautes grilles peintes en blanc. » (Carpentier 1962, 334-335)

Ce désenchantement atteint en fait toute une culture, la mémoire des lectures favorites du passé, des grandes œuvres des Lumières qu'Esteban perçoit désormais comme compromises par les violences et les injustices dont elles semblent avoir ouvert la voie. Dans la guerre des courses à laquelle Esteban, toujours sous la houlette de Victor Hugues, a participé, la remarque du commandant du navire était révélatrice : " Nous vivons dans un monde absurde. Avant la révolution ces îles étaient visitées par un bateau négrier qui appartenait à un armateur philosophe ami de Jean-Jacques. Et savez-vous comment il s'appelait? Le Contrat social. »

De retour à La Havane, examinant les livres qui, à la veille de son grand périple, ont nourri sa quête de la révolution, Esteban prend toute la mesure de son étrangeté aux principes et valeurs des Lumières qui sont cependant demeurées d'incontestables autorités intellectuelles, politiques et morales pour ses proches - et notamment pour Sofia - demeurés à Cuba : «Ces années lui semblaient courtes, maintenant qu'il les avait laissées derrière lui. Et cependant elles avaient eu le pouvoir de vieillir terriblement certaines choses, certains livres surtout. Une rencontre avec l'abbé Raynal, sur les rayons de la bibliothèque lui donna envie de rire. Le baron d'Holbach, Marmontel, avec ses Incas d'opéra-comique, le Voltaire des tragédies si subversivement actuelles il y avait à peine dix ans lui semblaient lointains, hors d'époque, aussi dépassés que pouvait l'être un traité de pharmacopée du $\mathrm{XIV}^{\mathrm{e}}$ siècle. Mais rien n'était aussi anachronique, aussi incroyablement fissuré, crevassé, amoindri par les événements que le Contrat social. Il ouvrit l'exemplaire dont les pages étaient pleines d'interjections admiratives, de gloses, de notes tracées par sa main, sa main de jadis. » (Carpentier 1962, 343-344)

Mais cette solitude d'Esteban ne tient pas qu'à l'abandon d'une culture philosophique et littéraire : sa fratrie - et pour l'essentiel Sofia - demeure attachée aux certitudes révolutionnaires. Quand il développe le récit de son «odyssée» devant sa famille retrouvée, Esteban est absolument incompris. Cette révolution avait répondu, certes, à un obscur élan millénaire qui aboutissait à l'aventure la plus ambitieuse de l'être humain. Mais Esteban était atterré par le coût de l'entreprise : «Nous oublions trop vite les morts. "Morts de Paris, de Lyon, de Nantes, d'Arras (et il accumulait les noms de cités qui révélaient maintenant l'étendue de leurs souffrances, comme Orange) ; morts des pontons de l'Atlantique, des camps de Cayenne, de tant d'autres lieux, sans oublier ceux dont le calcul devenait impossible, séquestrés, défenestrés, disparus ..., auxquels il fallait ajouter ces cadavres vivants qu'étaient les hommes à la vie brisée, à la vocation frustrée, à la tâche tronquée, qui traîneraient à jamais une vie lamentable, quand ils n'auraient pas eu l'énergie nécessaire pour se suicider. » (Carpentier 1962, 350).

L'incompréhension de Sofia est totale : «Elle admettait que les excès de la révolution étaient déplorables ; cependant les grandes conquêtes humaines ne s'obtenaient que par la douleur et le sacrifice. En somme, rien de grand ne se faisait sur terre sans effusion de sang. » (Carpentier 1962, 352) 
Esteban ne peut plus que constater son étrangeté absolue à l'espace qui fut le sien. Mais il paiera encore lourdement pour son passé révolutionnaire : arrêté sur ordre des autorités espagnoles, il sera déporté pour de longues années dans le bagne de Ceuta, possession espagnole du nord du Maroc. C'est par un chemin tout aussi difficile, tout aussi semé d'épreuves que Sofia connaîtra la même désillusion à l'égard de l'utopie révolutionnaire : Sofia rejoint Victor Hugues, son amant d'autrefois, devenu agent du Directoire en Guyane. Elle assiste là à la déferlante de la réaction : retour des prêtres réfractaires qui avaient refusé de prêter serment à la constitution révolutionnaire et enfin - et c'est là pour elle l'épreuve la plus dure qui la conduira à quitter Victor Hugues et à s'embarquer pour l'Europe - réinstauration violente du système esclavagiste : « (...) la loi du 30 floréal an X était promulguée, d'après laquelle on rétablissait l'esclavage dans les colonies françaises d'Amérique ; elle annulait les effets du décret du 16 pluviôse an II. Une immense allégresse éclata chez les possédants, planteurs et propriétaires terriens, promptement informés de ce qui les intéressait, si promptement que les messages avaient volé par-dessus les bateaux, on avait appris en outre qu'on reviendrait au système colonial antérieur à 1789, ce qui permettrait d'en finir une bonne fois avec les élucubrations humanitaires de cette sale révolution. À la Guadeloupe, à la Dominique, à Marie Galante, la nouvelle fut annoncée au milieu des salves et des illuminations, tandis que des milliers de « ci-devant » citoyens libres étaient à nouveau conduits à leurs anciens baraquements sous une avalanche de coups de bâton et de coups de fouet. Les Grands Blancs de jadis se précipitèrent à travers champs, suivis de meutes de chiens, à la recherche de leurs anciens esclaves, rendus au contre maître avec des chaînes au cou. » (Carpentier 1962, 426-427).

Sofia rejoint dès lors Esteban dans une commune désillusion à l'égard de la Révolution. Le départ de Sofia pour l'Europe, puis son installation à Madrid où elle réussit à obtenir la libération d'Esteban sont à l'origine de la réunion des deux protagonistes du roman mais aussi d'un épilogue tragique et aussi historiquement ancré que les séquences précédentes : la disparition du couple enfin réuni dans l'insurrection des madrilènes contre les troupes françaises de Napoléon le trois mai 1808. La dérive napoléonienne de la Révolution française - qui avait promis la liberté à l'humanité tout entière - atteint alors son paroxysme dans la «nuit du lugubre carnage, d'exécutions en masse, d'extermination près du Manzanarès et dans le quartier de la Moncloa », nuit des fusillades magistralement illustrée par Goya. Goya dont les légendes des gravures consacrées aux Désastres de la guerre sont citées en exergue de chaque chapitre du roman.

L'enquête finale menée par Carlos, le frère aîné venu de la Havane pour savoir ce qu'a été le sort des deux disparus, permet d'apprendre que Sofia a été la première à prendre les armes et à descendre dans les rues ensanglantées et qu'Esteban, lui-même armé, l'a suivie. Mais l'enquête menée non sans difficultés par Carlos suggère aussi ce qu'a été, dans cette phase finale l'évolution idéologique et culturelle des deux personnages et tout particulièrement d'Esteban. La rupture de ce dernier avec la littérature et la philosophie des Lumières s'est confirmée et est même devenue irréversible : «C'était maintenant au tour du libraire de parler : Esteban ne voulait rien savoir de philosophie, de travaux d'économistes, ni d'écrits qui traitassent de l'histoire de l'Europe pendant les dernières années. Il lisait des récits de voyages ; les poésies d'Ossian ; le roman des chagrins du jeune Werther ; de nouvelles traductions de Shakespeare. Et l'on se souvenait qu'il s'était enthousiasmé pour Le Génie du Christianisme, ouvrage qu'il qualifiait d'absolument extraordinaire » (...). (Carpentier 1962, 459)

Esteban était ainsi devenu, avant de disparaître à jamais dans la violence qui l'a suivi tout au long de sa vie un lecteur enthousiaste des œuvres majeures du premier romantisme. 


\section{CONCLUSION}

Le roman d'Alejo Carpentier, Le Siècle des Lumières, publié dès 1958, a en quelque sorte devancé d'une vingtaine d'années les analyses traitant de la même question - la désillusion à l'égard de l'imaginaire utopique et révolutionnaire. Analyses également ancrées - nous l'avons évoqué en nous référant aux recherches de Bronislaw Baczko et plus précisément à son ouvrage Le Siècle des Lumières - sur le XVIII ${ }^{\mathrm{e}}$ siècle des Lumières et de la Révolution française. Les analyses en question sont sans nul doute d'un autre ordre : elles relèvent d'une réflexion explicitement historique, ne mobilisent nullement la fiction et sont sous-tendues par une appréhension des grandes utopies meurtrières du $\mathrm{XX}^{\mathrm{e}}$ siècle et de leur démantèlement. Il importe toutefois de noter que pour Alejo Carpentier, comme pour Bronislaw Baczko, l'œuvre de Goya a été l'œuvre la plus suggestive. Le Goya des Désastres de la guerre bien sûr, mais peut-être plus encore le Goya des Peintures noires de la Maison du Sourd et plus précisément la peinture murale intitulée Asmodée ou Vision fantastique : deux hommes volants enlacés, enveloppés d'un grand manteau rouge, s'élèvent au-dessus d'une plaine où les hommes s'entretuent. Ils se dirigent vers une cité inondée de lumière, construite sur un mont escarpé qui paraît inaccessible. Une cité qui est peut-être la cité de la liberté, de la justice et du bonheur. Mais au bas du tableau, dans son angle droit, un soldat sans visage et armé d'un fusil met en joue les hommes volants. Le message est clair et c'est indéniablement le message du roman d'Alejo Carpentier : la marche vers l'utopie est une marche à la mort.

\section{REFERENCES}

Baczko, Bronislaw. Lumières de l'utopie. Paris, Payot, 1978.

Carpentier, Alejo. Le Siècle des Lumières. Traduit de l'espagnol par René, L.-F. Durand. Paris, Gallimard, 1962. Hamon, Philippe. « Pour un statut sémiologique du personnage ». Littérature, no. 6 (Mai), 1972, 86-110.

Morel, Anne-Rozenn. «L'utopie au service de l'action utopique », dans Temps, durée dans la littérature des Lumières et ses marges, études réunies par Jean Marie Goulemot, Paris, Le Manuscrit, coll. Réseau Lumières, 2010, p. 127-149.

Tatin-Gourier, Jean-Jacques. «Formes et temporalités du démantèlement des utopies dans Cleveland », dans Temps, durée dans la littérature des Lumières et ses marges, études réunies par Jean Marie Goulemot. Paris, Le Manuscrit, coll. Réseau Lumières, 2010, 107-127.

\section{REVOLUCIJA I UTOPIJA U KNJIZI VEK PROSVEĆENOSTI ALEHA KARPENTJERA: PUTEVIMA OTREŽNJENJA}

Od odjeka Francuske revolucije do Kariba - ukidanja i obnavljanja ropstva - pa sve do terora u revolucionarnoj Francuskoj i Španiji okupiranoj od strane Napoleonovih trupa, dvoje mladih ljudi, Sofija i Esteban, prolaze stazama ushićenja i oduševljenja do potpunog razočarenja i emotivnog rasula, pre nego što će nestati u Madridu u krvoproliću Trećeg maja, koje je Goja-često pominjan u romanuovekovečio. Putevi mladalačke utopije i nadanja završavaju se apokalipsom. Uglavnom se oslanjajući na radove istoričara društvenih predstava Bronislava Backa i baveći se transformacijom likova tokom sučeljavanja s dešavanjima u romanu, nastojaćemo da rasvetlimo tu svojevrsnu dinamiku izneverenih očekivanja.

Ključne reči: francuska revolucija, ropstvo, utopija, razočaranje, Francuska, ostrva 